Subscriber access provided by Caltech Library

\title{
Communication
}

\section{Tellurium: Fast Electrical and Atomic Transport along Weak Interaction Direction}

Yuanyue Liu, Wenzhuo Wu, and William A. Goddard

J. Am. Chem. Soc., Just Accepted Manuscript • DOI: 10.1021/jacs.7b09964 • Publication Date (Web): 22 Dec 2017

Downloaded from http://pubs.acs.org on December 22, 2017

\section{Just Accepted}

"Just Accepted" manuscripts have been peer-reviewed and accepted for publication. They are posted online prior to technical editing, formatting for publication and author proofing. The American Chemical Society provides "Just Accepted" as a free service to the research community to expedite the dissemination of scientific material as soon as possible after acceptance. "Just Accepted" manuscripts appear in full in PDF format accompanied by an HTML abstract. "Just Accepted" manuscripts have been fully peer reviewed, but should not be considered the official version of record. They are accessible to all readers and citable by the Digital Object Identifier (DOI®). "Just Accepted" is an optional service offered to authors. Therefore, the "Just Accepted" Web site may not include all articles that will be published in the journal. After a manuscript is technically edited and formatted, it will be removed from the "Just Accepted" Web site and published as an ASAP article. Note that technical editing may introduce minor changes to the manuscript text and/or graphics which could affect content, and all legal disclaimers and ethical guidelines that apply to the journal pertain. ACS cannot be held responsible for errors or consequences arising from the use of information contained in these "Just Accepted" manuscripts. 
Two-dimensional (2D) materials, such as graphene, $\mathrm{MoS}_{2}$, black phosphorus and hexagonal boron nitride, show great promise for many applications, including electronics, optoelectronics, energy conversion, and energy storage ${ }^{1}$. Recently, few-layer Te joins the family of $2 \mathrm{D}$ materials $\mathrm{s}^{2-7}$. It exhibits air-stable transistor performance comparable or superior to other $2 \mathrm{D}$ materials ${ }^{7}$, making Te attractive for nanoelectronics. Bulk Te exhibits interesting topological properties, such as transition from a trivial insulator to strong topological insulator (metal) under shear (hydrostatic or uniaxial) strain ${ }^{8}$, and presence of Weyl nodes ${ }^{9,10}$. Te consists of helical -Te-Te-Te- chains arranged parallel in a trigonal lattice. The intra-chain distance between neighboring Te atoms is $2.9 \AA$, while the inter-chain distance is $3.5 \AA$. Therefore, it has been assumed that the interaction along the chain is strong and covalent, while between the chains is weak. Some papers claim that the inter-chain interaction is van der Waals (vdW) type ${ }^{5,8,11-17}$. In anisotropic materials, the transport along weak interaction direction is usually much slower than that along chemical bond directions. For example, the electrical resistivity across the $\mathrm{MoS}_{2}$ (or graphite) layers is $\sim 10^{2}$ (or $10^{3}$ ) higher than that along the layer ${ }^{18,}$

\begin{abstract}
In anisotropic materials, the electrical and atomic transport along the weak interaction direction is usually much
slower than that along the chemical bond direction. However, Te, an important semiconductor comprised of helical atomic chains, exhibits nearly isotropic electrical transport between intra-chain
and inter-chain directions. Using first-principles calculations to study the bulk and few-layer Te, we show that this isotropy is related with similar effective mass and potential for charge carri-
ers along different transport directions, benefiting from the delocalization of the lone-pair electrons. This delocalization also enhances the inter-chain binding, although it is still significantly
weaker than the covalent intra-chain bonding. Moreover, we find a fast diffusion of vacancies and interstitial atoms along and across the chains, enabling rapid self-healing of these defects at
room temperature. Interestingly, the interstitial atoms diffuse along the chain via a concerted-rotation mechanism. Our work reveals the unconventional properties underlying the superior
performance of $\mathrm{Te}$, while providing insight into the transport in anisotropic materials.
\end{abstract}

${ }^{19}$. Hence, one would expect a similarly slower charge transport across the Te chains than that along the chain. However, experiments have shown that the electrical conductance along the chain is only $\sim 1.13$ (for few layer ${ }^{7}$ ) or $1.41 \pm 0.11$ (for bulk ${ }^{20}$ ) times higher than that across the chains. This surprising behavior calls for a deeper understanding on the nature of the inter-chain interaction and the transport properties.

Here we perform first-principles calculations using the Vienna Ab-initio Simulation Package (VASP) ${ }^{21,22}$ with projector augmented wave (PAW) pseudopotentials ${ }^{23,}{ }^{24}$. The Perdew-BurkeErnzerhof (PBE) exchange-correlation functional ${ }^{25}$ is used to relax the systems, and obtain the positions of band edges and the effective masses. The HSE functional ${ }^{26}$ is used to extract more accurate values of band gaps and band edge energies. When calculating the binding/exfoliation energy, we also include the empirical D3 correction ${ }^{27}$ to account for London Dispersion interactions that are not included in PBE. More details can be found in the Supporting Information (SI).

We first study few-layer Te, which is more technologically important for nanoelectronics than the bulk material. Few-layer Te is terminated by the lowest energy surfaces $10-10,{ }^{7}$ which has a rectangular primitive cell. Fig. la shows the atomic structure of a 4-layer Te as an example, where the y direction is along the chain (helical axis), and the $\mathrm{x}$ is normal to chain and parallel to the surface. The distances between $\mathrm{Te}$ atoms decrease as the material becomes thinner (see Table S1 for lattice parameters of few-layer Te with different thickness). Specifically, the intra-chain and inter-chain distances are $2.9 \AA$ and $3.51 \AA$ (anisotropy ratio: 1.21 ) in the bulk, and shrink to 2.87 and 3.37 (1.17) in the bilayer, then to 2.78 and 3.04 (1.09) in monolayer. This abrupt reduction of the distances in monolayer Te indicates a significant structural reconstruction, and the small anisotropy ratio suggests the formation of strong covalency in the inter-chain interactions (see Fig. S1; the same structure has been reported in other papers $\left.{ }^{2-4}\right)$. The origin of this reconstruction and its impact on the electronic properties will be discussed below. 

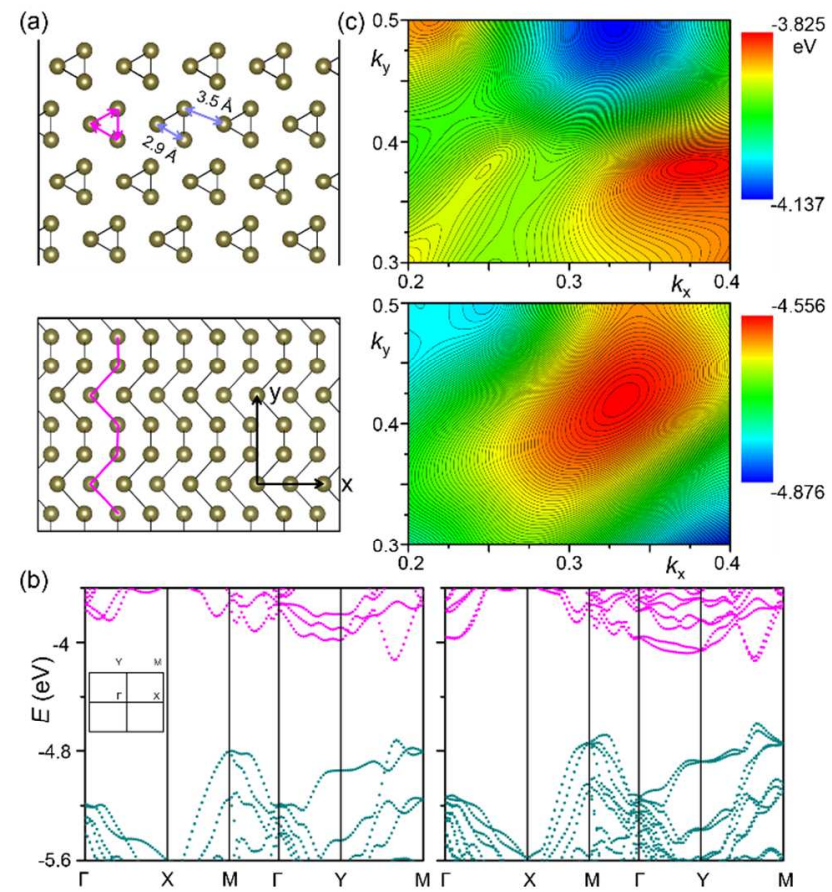

Fig. 1. (a) Atomic structure of 4 layer Te. Top: side view; Bottom: top view. Only the surface and sub-surface layers are visible in the top view. The pink lines highlight the sub-surface layer. The $\mathrm{x}$ direction is across the chains and parallel to the surfaces, while $\mathrm{y}$ is along the chain. The blue arrows highlight the intrachain and inter-chain distances. (b) Electronic band structure of 4 layer Te, calculated by using PBE functional. Left: without spinorbit coupling (SOC); Right, with SOC. The valence bands are shown in cyan and the conductions bands are in pink. The energies are referred to the vacuum level. The first Brillouin zone is shown in the inset with high symmetry points marked. If the chains are non-interacting, then flat bands would appear in $\Gamma-\mathrm{X}$ and Y-M regions as the electrons/holes can only transport along the chain. (c) Contour plots of the bottom conduction band (top) and the top valance band (bottom). The axes are in the units of the reciprocal lattice vectors.

Fig. $1 \mathrm{~b}$ shows the band structure of a 4-layer Te, calculated using PBE functional. The effect of the spin-orbit coupling is notable, resulting in a splitting of the doubly-degenerate band edge states (i.e. valence band maximum (VBM) and conduction band minimum (CBM)), and a smaller band gap. Fig. 1c shows that the band gap is indirect, with VBM and CBM located off the highsymmetry points. The constant-energy contours near the band edges show a ellipse shape with principle axes tilted from $\mathrm{x}$ and $\mathrm{y}$.

The indirect nature of the band gap is also observed in other multi-layers. As shown in Fig. 2a, the positions of VBM and CBM change linearly as a function of $1 / n$ or $1 / d$, where $n$ is the number of layers, and $d$ is the thickness which can be related by using the "thickness" of Te layer in the bulk: $d=n * 3.91 \AA$. The slope and intercept are listed in Fig. 2. The slope is a measure of the change of the properties with thickness, and the intercept is relevant for the bulk material. The band gap (calculated using the HSE functional) decreases as thickness increases, also following a linear relation with $1 / n$ or $1 / d$, and becomes $0.4 \mathrm{eV}$ for infinitelythick (bulk) Te, close to the experimental value $0.35 \mathrm{eV}^{28}$. The $\mathrm{VBM} / \mathrm{CBM}$ energy (calculated using the HSE functional) also decreases/increases linearly with $1 / n$ or $1 / d$, as shown in Fig. 2c. Note that the VBM energies are relatively high $(-5--4.67 \mathrm{eV})$ compared with some of the commonly used electrode metals (Pt: 5.12 - -5.93, Pd: -5.22 - -5.60, Au: -5.10 - -5.47, Ni: $-5.04-$ $5.35, \mathrm{Co}:-5)^{19}$, which suggests a lower Schottky barrier for hole injection than for electron ${ }^{29-31}$. This could be one reason why Te is p-type ${ }^{7}$. The effective masses of electrons and holes transporting across the chains $\left(m_{\mathrm{e}-\mathrm{x}}\right.$ and $\left.m_{\mathrm{h}-\mathrm{x}}\right)$ increases linearly with $1 / n$ or $1 / d$. The extrapolated value of $m_{\mathrm{h}-\mathrm{x}}$ for bulk Te is $0.07 m_{0}$, close to the experimental value $0.108 \mathrm{~m}_{0} .{ }^{28}$ It is interesting that the electrons transporting along the chain are heavier than those across the chains (i.e. $m_{\mathrm{e}-\mathrm{y}}>m_{\mathrm{e}-\mathrm{x}}$ ). Although $m_{\mathrm{h}-\mathrm{y}}<m_{\mathrm{h}-\mathrm{x}}$ for $\mathrm{n}<6$, they quickly approach each other, and reverse the order for thick samples and bulk $\mathrm{Te}^{28}$. Overall, the $m_{\mathrm{h}}$ and $m_{\mathrm{h}}$ of few-layer Te are comparable or even smaller than common 2D semiconductors $\left(\mathrm{MoS}_{2}: \sim 0.5 \mathrm{~m}_{0}{ }^{32}\right.$ black phosphorus: $\sim 0.1 \mathrm{~m}_{0}^{33}$ ), which is one reason for its high performance observed experimentally.
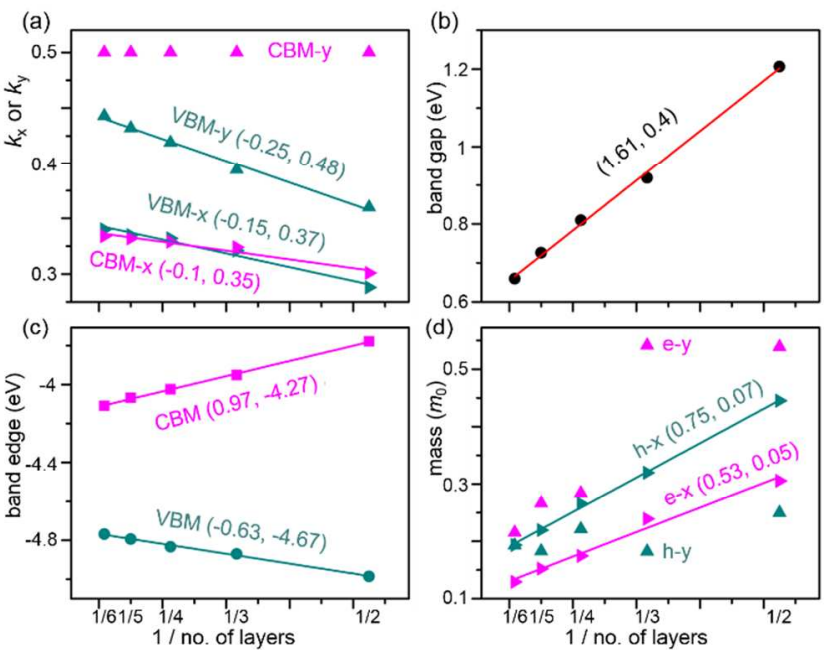

Fig. 2. Electronic properties of Te as a function of the thickness. The lines are linear fits, with slopes and interceptions shown in the corresponding brackets. (a) Positions of the Valence Band Maximum and the Conduction Band Minimum (CBM), in the units of the reciprocal lattice vectors. (b) Band gaps. (c) Band edge energies, with respect to vacuum level. (d) Effective masses of electrons and holes. (b)-(c) are calculated by using HSE functional, while (a) and (d) are by PBE functional. The electronic properties of monolayer Te differ significantly from few layer, and the corresponding values are shown in the text.

These linear trends can be used to estimate the electronic properties of Te at given thickness. However, the monolayer Te does not fall into these trends. It has a direct band gap of $1.34 \mathrm{eV}$ located at $\Gamma$ point, significantly different from the extrapolated value $2 \mathrm{eV}$. Similarly, the VBM and CBM energies (-5 and -3.66 $\mathrm{eV})$, and effective masses $\left(m_{\mathrm{e}-\mathrm{y}}: 0.76 m_{0} ; m_{\mathrm{e}-\mathrm{x}}: 0.15 ; m_{\mathrm{h}-\mathrm{y}}: 0.3 ; m_{\mathrm{h}-}\right.$ $x: 0.11$ ), also differ substantially from the linear extrapolations. These deviations can be attributed to the significant structural reconstruction mentioned above. We point out that the monolayer Te has several polymorphs, which have been studied in other work $^{2,4}$.

In addition to the similarity of effective masses along different directions in few-layer and bulk Te, we find that the potential distribution is also nearly-isotropic, which is in sharp contrast with common $\mathrm{vdW}$ materials. Fig. 3a plots the exchangecorrelation potential in bulk Te, plotted along or normal to the chain/layer. The oscillations along different directions have similar amplitude. In contrast, common vdW materials, such as $\mathrm{MoS}_{2}$, have much stronger oscillation along the $\mathrm{vdW}$ direction (perpendicular to the layer) than along the chemical bonds (Fig. 3b). This indicates that the charge carriers in Te experience a more isotropic potential during transport than those in common vdW materials. 

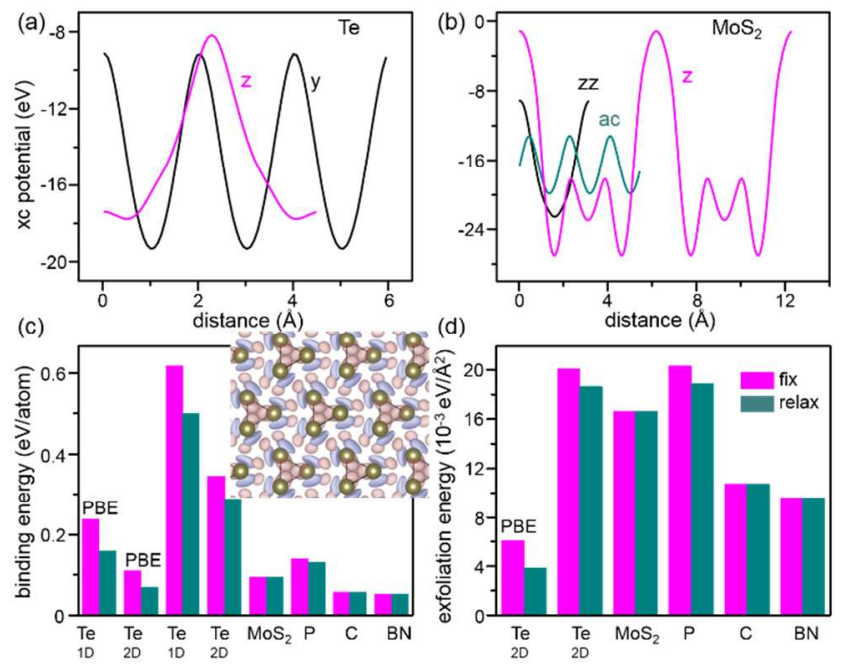

Fig. 3. (a) Exchange-correlation potential of bulk Te, plotted along the chain (y), and perpendicular to the layer (z). The potential is averaged over the planes normal to the plotting direction. The zero of distance is set to an arbitrary point in the primitive cell. (b) Potential of $\mathrm{MoS}_{2}$, plotted along the zigzag (zz) direction, armchair (ac) direction, and perpendicular to the layer (z). (c) Binding energies of Te chain (1D), Te layer (2D), and component units of other vdW materials (P: black phosphorus; C: graphene; $\mathrm{BN}$ : hexagonal boron nitride) in their bulk. The inset shows the charge density redistribution when Te chains are bound together. Blue iso-surface: electron depletion; Red: electron accumulation. (d) Exfoliation energies (binding energies normalized by the surface area). "PBE" means the values are calculated by using PBE functional only, while the others include D3 correction. "Fix" means the atomic coordinates of layers/chains are fixed to those in the bulk, and "relax" means they are fully relaxed in vacuum.

This sharp difference motivates us to investigate the nature of the inter-chain interaction. Fig. $3 c$ inset shows the charge density redistribution when $\mathrm{Te}$ chains are bound together. The lone-pair electrons are delocalized by depleting the density in their original positions and enhancing the density in the inter-chain region, which adds "metallic" bonding character into the inter-chain interaction. The delocalization of Te lone-pair electrons lowers the effective mass and changes the potential in the inter-chain region, and hence enhances the transport across the chains. This delocalization can be explained by the relatively weak nucleus attraction compared with other elements in the same group before Te (i.e., $\mathrm{O}, \mathrm{S}$, and $\mathrm{Se}$ ). The nucleus attraction becomes weaker when moving down the periodic table, which makes the element after Te, i.e. Po, a metal with completely delocalized electrons.

The delocalization of Te lone-pair electrons also enhances the inter-chain interaction. We calculate the inter-chain/layer binding energy (Fig. 3c) as:

$$
E_{\text {bind }}=\left(E_{\text {chain/layer }}-E_{\text {bulk }}\right) / n_{\text {atom }} \text {. }
$$

where $E_{\text {bulk }}$ is the energy of the bulk material, $E_{\text {chain/layer }}$ is the energy of isolated chain/layer (which can be fixed to the same atomic coordinates as in the bulk, or fully relaxed), and $n_{\text {atom }}$ is the number of atoms. It is well known that the PBE functional does not describe well the vdW interaction, and gives an underestimated value $\sim 0.01 \mathrm{eV} /$ atom for common $\mathrm{vdW}$ materials $\left(\mathrm{MoS}_{2}\right.$, black phosphorus, graphene, and hexagonal boron nitride). However, PBE predicts a large $E_{\text {bind }}, 0.07$ (relaxed) -0.11 (fixed) eV/atom for Te layer, and $0.16-0.24 \mathrm{eV} /$ atom for $\mathrm{Te}$ chain. The underestimated $\mathrm{vdW}$ interaction in PBE calculation can be corrected by using D3 approach, which gives an $E_{\text {bind }}$ for Te chain/layer several times stronger than that in common vdW materials. Given that the $E_{\text {bind }}$ for chains and layers are compara- ble, the Te is best viewed as consisting of strongly coupled chains. Note that in bulk Te, each chain is bound with six other chains; however, for monolayer Te, it is bound with only two chains. The reduction of coordination enhances the inter-chain binding in monolayer Te, resulting in a significant shortening of the inter-chain distance. Although the inter-chain interaction in bulk and multilayer Te is stronger than common vdW, it is still significantly weaker than the intra-chain bonding $(2.34 \mathrm{eV} /$ atom, calculated using PBE; see the SI for the Young's modulus).

Interestingly, although the $E_{\text {bind }}$ of Te is several times larger than that of common vdW materials, the exfoliation energy, which is the interlayer binding energy normalized by the surface area, is comparable (Fig. 3d): 1.1-1.2 than $\mathrm{MoS}_{2}, 0.99$ of black phosphorus, 1.7-1.9 than graphene, and 1.9-2.1 than hexagonal boron nitride. This is because of the low density of atoms per surface area in Te, being 0.31-0.45 of the others. The comparable exfoliation energy suggests that the Te can be exfoliated into thin layers like common vdW materials, despite its different binding nature.

We also find a fast atomic transport across the chains, such as diffusion of vacancies and interstitial atoms. As shown in Fig. 4, when the vacancy (or a counter-atom) hops from one chain to another, the counter-atom in the inter-chain region forms a bond with one chain (bond length $2.88 \AA$, similar to that in the perfect material $2.9 \AA$ ), and is further coupled with the atom from other layer with a distance of only $3.15 \AA$. These effects help stabilize the transition state, leading to a low barrier $E_{\mathrm{b}} \sim 0.7 \mathrm{eV}$. In contrast, the vacancy diffusion across the layers in common vdW materials encounters a large $E_{\mathrm{b}}$ (e.g., $E_{\mathrm{b}}>7 \mathrm{eV}$ for graphite). This is because of the relatively strong inter-chain interaction in Te, which results in an inter-chain distance not far from the bonding length ( 3.51 vs 2.9 , ratio: 1.21$)$. Therefore, the counter-atom can still form the bond or effectively couple with the chain even in the inter-chain region. However, in graphene, the interlayer distance is too large compared with the bond length of C (3.47 vs 1.43 , ratio: 2.43 ), hence the diffusing atom in the interlayer region cannot form bonds or effectively couple with the layers, leading to a large $E_{\mathrm{b}}$. Similarly, when an interstitial atom hops from one chain to another, it also forms bonds/couples with the chains, thus the $E_{\mathrm{b}}$ is low $0.77 \mathrm{eV}$.

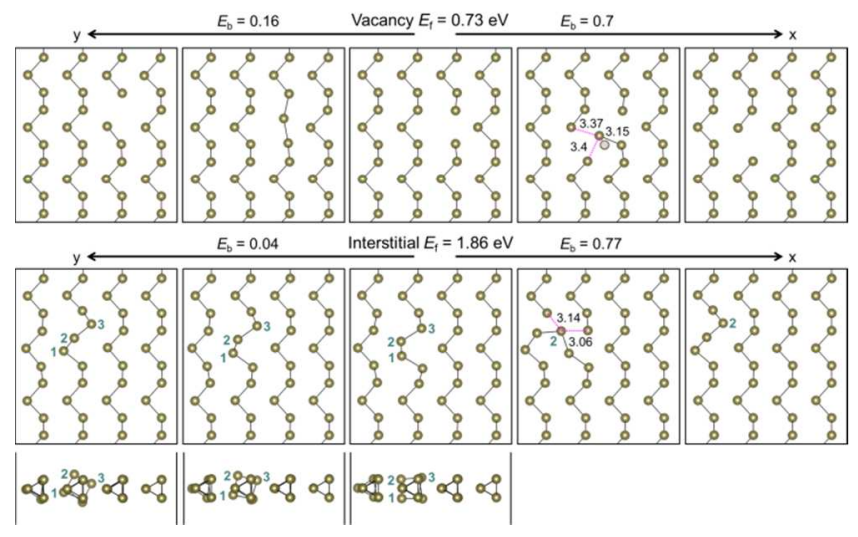

Fig. 4. Atomic structures of mono-vacancy (top) and interstitial atom (bottom), diffusing along the chain (y) and across the chains (x) of bulk Te. $E_{\mathrm{f}}$ stands for the formation energy of the defect, and $E_{\mathrm{b}}$ is the diffusion barrier. The transition states are shown in the middle panel of each diffusion path. For clarity, we show only one layer, except for the transition state of vacancy diffusing across the chains, in which case the atom marked in gray is from other layer. For the interstitial diffusing along the chain, we also show the side views to highlight the concerted-rotation mechanism. 
Moreover, we find that atomic transport along the chain has an even lower barrier. As shown in Fig. 4, for vacancy diffusion, the counter-atom stretches its original bond and forms a new bond with other atom at the transition state, giving an $E_{\mathrm{b}}$ of $0.16 \mathrm{eV}$, lower than that of common vdW materials ${ }^{34}$. Interestingly, diffusion of the interstitial atom along the chain involves the concerted motion of three atoms, as labeled in Fig. 4. At the initial state, the atom "2" can be viewed as the interstitial, which pushes the neighboring atom " 1 " away from its original position. At the transition state, the " 1 " restores its position, and pushes " 2 " and further " 3 " forward along the helix. At the final state, " 3 " is off its original position with " 2 " being the interstitial. When viewed along the chain, the diffusion looks like the collective rotation of the three atoms. The next periodic step would be " 2 " takes the original position of " 3 " and " 3 " becomes the new interstitial. During these processes, the coordination of atoms does not change (unlike diffusion in many other materials), hence the $E_{\mathrm{b}}$ is rather low $0.04 \mathrm{eV}$.

The hopping rate $w$ can be estimated by using $v^{*} \exp \left(-E_{\mathrm{b}} / k_{\mathrm{B}} T\right)$, where $k_{\mathrm{B}}$ is the Boltzmann constant, $T$ is the temperature, and $v$ is the vibration frequency typically $\sim 10^{13}$ per second. At room temperature, $w=1.7 * 10^{10}$ and $2 * 10^{12} \mathrm{~s}^{-1}$ for the vacancy and interstitial diffusion along the chain respectively. Thus these defects would quickly transport to the edges to reduce the energy by $E_{\mathrm{f}}$, which is the formation energy of the defect with respect to the bulk Te $(0.73 \mathrm{eV}$ for vacancy and 1.86 for interstitial). The diffusion across the chains is slower, $w \sim 6.9$ and $0.4 \mathrm{~s}^{-1}$ for the vacancy and interstitial respectively. These modes can be expedited by increasing the $T$. For example, at $400 \mathrm{~K}$ (note that the melting point is $723 \mathrm{~K}), w=7.6 * 10^{3}$ (vacancy) and $9.3 * 10^{2}$ (interstitial). These modes are not available in common vdW materials due to the large $E_{\mathrm{b}}$ as discussed early.

In summary, we show that the nearly isotropic electrical transport in chemically anisotropic Te is related with the similar effective mass and potential of charge carriers along different transport directions, benefiting from the delocalization of lonepair electrons. This delocalization also enhances the inter-chain interaction, although it is still significantly weaker than the intrachain bonding. We also find a fast transport of vacancies and interstitial atoms across the Te chains, which together with the fast intra-chain transport, enable a rapid healing of these defects at room temperature. Moreover, a novel concerted-rotation mechanism is revealed for interstitial atoms diffusing along the chain. Our work reveals the unconventional properties underlying the superior performance of $\mathrm{Te}$, and provides insight into the transport in anisotropic materials.

\section{ASSOCIATED CONTENT}

\section{Supporting Information}

Computation details; lattice parameters; discussion on the Young's modulus; atomic coordinates of 4 layer Te, monolayer Te, vacancies and interstitials diffusing along and across the chains (with corresponding energies);

\section{AUTHOR INFORMATION}

\section{Corresponding Author}

yuanyue.liu.microman@gmail.com orwag@caltech.edu Notes

The authors declare no competing financial interests.

\section{ACKNOWLEDGMENT}

Y. L. acknowledges the startup support from UT Austin. W. A. G. acknowledges the support by DOE CMS DE-SC0014607. This work used computational resources sponsored by the DOE's Office of Energy Efficiency and Renewable Energy and located at the National Renewable Energy Laboratory, and the Texas Advanced Computing Center (TACC) at UT Austin. W. Z. W. acknowledges the College of Engineering and School of Industrial Engineering at Purdue University for the startup support. W. Z. W. was partially supported by a grant from the Oak Ridge Associated Universities (ORAU) Junior Faculty Enhancement Award Program.

\section{REFERENCE}

1. Tan, C.; Cao, X.; Wu, X.-J.; He, Q.; Yang, J.; Zhang, X.; Chen, J.; Zhao, W.; Han, S.; Nam, G.-H.; Sindoro, M.; Zhang, H. Chemical Reviews 2017, 117, (9), 6225-6331.

2. Xian, L.; Paz, A. P.; Bianco, E.; Ajayan, P. M.; Rubio, A. $2 D$ Materials 2017, 4, 041003 .

3. Zhu, Z.; Cai, X.; Niu, C.; Wang, C.; Sun, Q.; Han, X.; Guo, Z.; Jia, Y. arXiv:1605.03253 2016

4. $\quad$ Zhu, Z.; Cai, X.; Yi, S.; Chen, J.; Dai, Y.; Niu, C.; Guo, Z.; Xie, M.; Liu, F.; Cho, J.-H.; Jia, Y.; Zhang, Z. Phys. Rev. Lett. 2017, 119, 106101 .

5. Du, Y.; Qiu, G.; Wang, Y.; Si, M.; Xu, X.; Wu, W.; Ye, P. D. Nano Lett. 2017, 17, (6), 3965-3973.

6. Huang, X.; Guan, J.; Lin, Z.; Liu, B.; Xing, S.; Wang, W.; Guo, J. Nano Lett. 2017, 17, 4619-4623.

7. Wang, Y.; Qiu, G.; Wang, Q.; Liu, Y.; Du, Y.; Wang, R.; III, W. A. G.; Kim, M. J.; Ye, P. D.; Wu, W. arXiv:1704.06202 2017.

8. Agapito, L. A.; Kioussis, N.; Goddard, W. A.; Ong, N. P. Phys Rev. Lett. 2013, 110, (17), 176401.

9. Hirayama, M.; Okugawa, R.; Ishibashi, S.; Murakami, S.; Miyake, T. Phys. Rev. Lett. 2015, 114, (20), 206401.

10. Tsirkin, S. S.; Souza, I.; Vanderbilt, D. Phys. Rev. B 2017, 96, (4), 045102.

11. Xia, Y.; Yang, P.; Sun, Y.; Wu, Y.; Mayers, B.; Gates, B.; Yin, Y.; Kim, F.; Yan, H. Adv. Mater. 2003, 15, (5), 353-389.

12. Wang, Q.; Safdar, M.; Xu, K.; Mirza, M.; Wang, Z.; He, J. ACS Nano 2014, 8, (7), 7497-7505.

13. Blum, F. A.; Deaton, B. C. Phys. Rev. 1965, 137, (5A), A1410A1417.

14. Chen, J.; Dai, Y.; Ma, Y.; Dai, X.; Ho, W.; Xie, M. Nanoscale 2017, 9, (41), 15945-15948.

15. Mayers, B.; Xia, Y. J. Mater. Chem. 2002, 12, (6), 1875-1881.

16. Epstein, A. S.; Fritzsche, H.; Lark-Horovitz, K. Phys. Rev. 1957, 107, (2), 412-419.

17. Li, P.; Sau, J. D.; Appelbaum, I. Phys. Rev. B 2017, 96, (11), 115446.

18. Kam, K.-K. Retrospective Theses and Dissertations (Iowa State University) 1982, Paper 8356.

19. CRC Handbook of Chemistry and Physics. $97 \mathrm{ed}$.

20. Skadron, P.; Johnson, V. A. Journal of Applied Physics 1966, $37,1912$.

21. Kresse, G.; Hafner, J. Phys. Rev. B 1993, 47, (1), 558-561.

22. Kresse, G.; Furthmüller, J. Phys. Rev. B 1996, 54, (16), 11169-

11186.

23. Kresse, G.; Joubert, D. Phys. Rev. B 1999, 59, (3), 1758-1775.

24. Blöchl, P. E. Phys. Rev. B 1994, 50, (24), 17953-17979.

25. Perdew, J. P.; Burke, K.; Ernzerhof, M. Phys. Rev. Lett. 1996, 77, (18), 3865-3868.

26. Paier, J.; Marsman, M.; Hummer, K.; Kresse, G.; Gerber, I. C.; Ángyán, J. G. J. Chem. Phys. 2006, 124, (15), 154709.

27. Grimme, S.; Antony, J.; Ehrlich, S.; Krieg, H. J. Chem. Phys. 2010, 132, (15), 154104.

28. Peng, H.; Kioussis, N.; Snyder, G. J. Phys. Rev. B 2014, 89, (19), 195206.

29. Liu, Y.; Stradins, P.; Wei, S.-H. Science Advances 2016, 2, (4), e1600069.

30. Liu, Y.; Xiao, H.; Goddard, W. A. J. Am. Chem. Soc. 2016, 138, (49), 15853-15856.

31. Kang, J.; Liu, W.; Sarkar, D.; Jena, D.; Banerjee, K. Phys. Rev. $X$ 2014, 4, (3), 031005.

32. Peelaers, H.; Van de Walle, C. G. Phys. Rev. B 2012, 86, (24), 241401.

33. Qiao, J.; Kong, X.; Hu, Z.-X.; Yang, F.; Ji, W. 2014, 5, 4475.

34. Cai, Y.; Ke, Q.; Zhang, G.; Yakobson, B. I.; Zhang, Y.-W. J. Am. Chem. Soc. 2016, 138, (32), 10199-10206. 
TOC

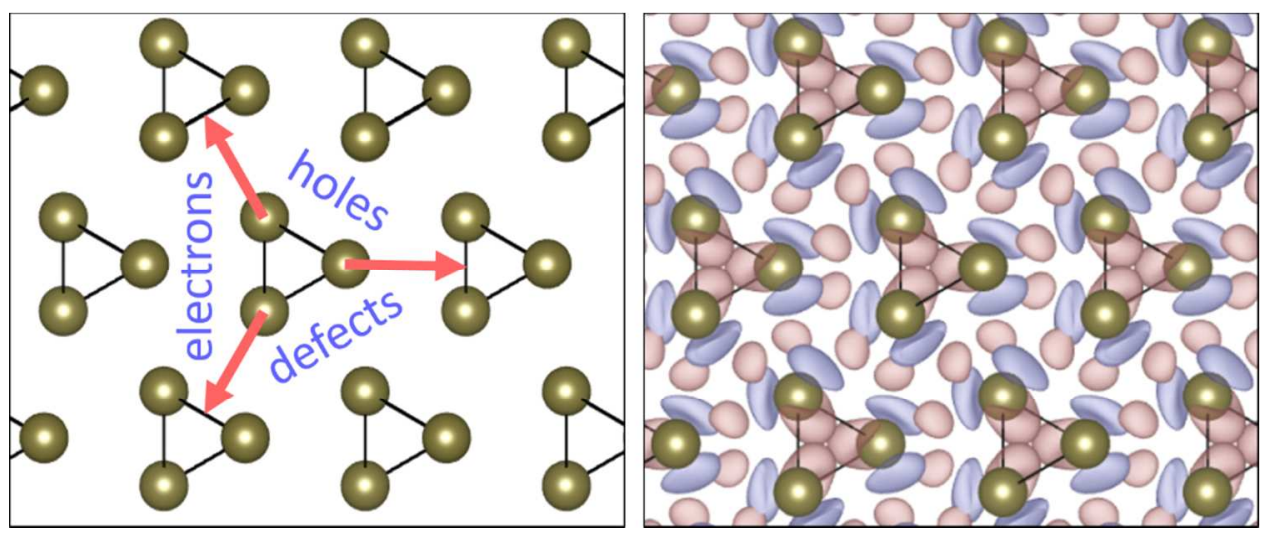

15

16 